\title{
Stochastic processes in a discrete model of ground combat
}

\author{
$\underline{\text { T. A. McLennan-Smith }}^{\mathrm{a}}$, M. Nelson ${ }^{\mathrm{b}}$, Z. Jovanoski ${ }^{\mathrm{a}}$, M. Rodrigo ${ }^{\mathrm{b}}$ and H. S. Sidhu $^{\mathrm{a}}$ \\ ${ }^{\text {a }}$ School of Science, UNSW, Canberra, ACT 2610, Australia \\ ${ }^{\mathrm{b}}$ University of Wollongong, Wollongong, NSW 2522, Australia \\ Email: t.mclennan-smith@adfa.edu.au
}

\begin{abstract}
Discrete models of combat are a rare part of the combat modelling literature. Our work introduces a stochastic version of a discrete ground combat based on Epstein theory featuring two adversarial sides, namely an attacker and a defender. Noticeably, the Epstein model of ground combat features an evolving battle front through a withdrawal mechanism to capture the connection between attrition and movement of the front historically prevalent in ground war. Our extension from the deterministic setting of the Epstein model to the stochastic setting is achieved by taking the exchange ratio of attackers lost to defenders to be a mean-reverting stochastic process. The extension of the exchange ratio to a stochastic process is interrupted to be the result of changing strategies and engagements by either side as well of the generally uncertainty of warfare known as the "Fog of War" upon the outcome of combat.

In the deterministic setting of our model, our toy numerical example results in an attacker victory. In the extension of the exchange ratio to a stochastic process, the attackers are no longer assured victory. However, the variations in the exchange ratio can be of benefit to the attackers in that they may achieve victory in a shorter combat duration and as a consequence suffer less attrition. Thus we interpret the stochastic process as introducing a "risk vs reward" scenario for the attackers where the risk is quantified through the volatility of the process. Our numerical simulations explore the shift in the outcome of combat for the attackers as they take on additional risk and more uncertainty is introduced into combat.

We observe the probability that the attacker is victorious, the time till victory when the attacker is victorious, and the remaining ground force strength of the attacking forces for varying volatility. Our results show that for increasing values of the volatility of the exchange ratio process, the probability of an attacker victory increases but the combat duration decreases and the remaining combat power of the attacker forces increases.
\end{abstract}

Keywords: Combat models, stochastic processes, discrete models 


\section{INTRODUCTION}

Battlefield simulation models have traditionally been based around the use of deterministic models. These have often taken the form of systems of differential equations that are ultimately based upon the classical Lanchester theory of combat [Lancaster, 1916]. The feature of any deterministic model is that the eventual outcome of the battle is pre-determined once the initial conditions are set.

Such models do not capture the "Fog of War" — the uncertainty in military operations [Clausewitz and Maude 1982; Herbig, 1986; Waldman, 2010|. One way to allow for uncertainty is to utilise stochastic models. These allow for random variations in one or more inputs over time. The outcome of the engagement is no longer uniquely determined by the initial conditions, rather there is a probability distribution describing the potential outcomes. Stochastic models therefore provide a more realistic representation of combat dynamics, albeit at the expense of greater computational effort. Depending upon the underlying model parameters and the size of the stochasticity in the parameters the predictions of the battle may be robust, i.e. predictability with a high degree of certainty and precision, or sensitive to numerous parameters and initial conditions. Advanced knowledge of the robustness of the model dynamics is crucial to assist central command in their decision making so as to seize the initiative on the field of battle.

This paper investigates a stochastic battlefield model in the form of difference equations. The original model, using deterministic differential equations, was proposed by Epstein [1985] and has been used to simulate battles between the United States and the Soviet Union in the Middle East [Epstein, 1987]. Sedaghat [2002] developed a version of Epstein's model that uses deterministic difference equations without air support, i.e. it is a ground model. Sedaghat [2002| showed that the deterministic model exhibits a range of behaviours including convergence to a fixed point, oscillations, and chaos.

Buontempo et al. [2006| note that Epstein's model can result in unphysical trajectories; in some circumstances the attrition rates can exceed $100 \%$. They also suggest that the circumstances under which either the attacker ceases combat or the defender fully withdraws are too restrictive. To overcome these defects they developed a new model which builds upon Epstein's concept of adaptive dynamics. The model is symmetric with respect to the two forces, so that the roles of attacker and defender may switch during battle, and produces results that are always physically correct. Finally, parameters are introduced which provide a framework for incorporating the internal structure and dynamics of the two forces. attempt to include sociological factors. For example, some parameters can be interpreted as taking into account qualities such as culture, leadership, dedication to the mission, the strength of command, and control structure.

\section{THE STOCHASTIC COMBAT MODEL}

In our model formulation we consider two ground forces, an attacker and a defender, engaged in combat where each side is able to undergo adaptive behaviour during the course of combat. Specifically, the defenders are able to incorporate a withdrawal strategy in order to mitigate their attrition once a threshold value is crossed. The attackers are able to increase their own attrition rate in order to push the ground combat forward with the intention of forcing the defenders to withdraw. The dynamics of the combat power of the attacker and defender ground forces on day $n+1$, denoted by $A_{n+1}$ and $D_{n+1}$ respectively, are given by

$$
\begin{aligned}
& A_{n+1}=\left(1-\alpha_{n}\right) A_{n}, \\
& D_{n+1}=\left(1-\delta_{n}\right) D_{n},
\end{aligned}
$$

where $A_{n}$ and $D_{n}$ are the respective combat powers of the forces on day $n, \alpha_{n}$ is the attacker attrition rate on day $n$, and $\delta_{n}$ is the defender attrition rate on day $n$. In a similar manner to Epstein [1985] and Sedaghat [2002], we define the attacker attrition rate on day $n$ through the relationship

$$
\alpha_{n}=\gamma_{n}\left(1-\frac{W_{n}}{W_{\max }}\right), \quad \alpha_{0} \in(0, a]
$$

where $\gamma_{n}$ is the prosecution rate of the attacker day $n, W_{n}$ is defender's withdrawal rate on day $n, W_{\max }$ is the defender's maximum withdrawal rate, and $a \in(0,1)$ is the attacker's prescribed attrition rate threshold. The prosecution rate of the attacker, $\gamma_{n}$, is defined by Epstein |1985| as the attrition rate at which the attacker is prepared to suffer in order to press the ground combat at its chosen pace. This rate is given by 
T. A. McLennan-Smith et al., Stochastic processes in a discrete model of ground combat

$$
\gamma_{n+1}=\gamma_{n}+\frac{1}{a}\left(a-\gamma_{n}\right) \cdot\left(a-\alpha_{n}\right), \quad \gamma_{0} \in(0, a]
$$

The defender's withdrawal rate is dependent upon wherever their attrition rate $\delta_{n}$ reaches the threshold value $d$, where $d \in(0,1)$ is the defenders prescribed attrition rate threshold. The defender's withdrawal rate on day $n+1$ is given by

$$
W_{n+1}=\left\{\begin{array}{cl}
W_{n}+\frac{W_{\max }-W_{n}}{1-d} \cdot\left(\delta_{n}-d\right) & , \delta_{n} \geq d, \\
0, & , \delta_{n}<d .
\end{array} \quad W_{0}=0 .\right.
$$

The defenders attrition rate on day $n$ is defined through the relationship

$$
\delta_{n}=\frac{\alpha_{n} A_{n}}{\rho_{n}^{+} D_{n}}
$$

where $\rho_{n}^{+}$is the ratio of attackers lost to defenders lost. In our model formulation, we take $\rho_{n}^{+}$to be a discrete, reflecting stochastic process on the domain $\bar{D}=[0, \infty)$ which is defined by

$$
\rho_{n}^{+}= \begin{cases}\rho_{n} & \text { for } n \in[0,1, \ldots N] \\ \rho_{n}-\inf \left\{\rho_{s}-L_{\rho}: s \in[N, N+1, \ldots, n]\right\} & \text { for } n>N\end{cases}
$$

where $N=\inf \left\{n>0: \rho_{n}<L_{\rho}\right\}, L_{\rho}>0$ is the lower bound on the exchange ratio, and $\rho_{n}$ is the meanreverting stochastic process defined by

$$
\rho_{n+1}=\rho_{n}+\lambda\left(\rho-\rho_{n}\right)+\sigma X_{n}, \quad \rho_{0}=\rho .
$$

Here $\rho$ is the mean level of the exchange ratio, $\sigma>0$ is the volatility of the process, $\lambda$ is the speed of mean reversion, and the variables $X$ are independent and identically distributed (i.i.d) normal distributed random variables where $X_{n} \sim \mathcal{N}(0,1)$. In our model, we interpret the stochastic nature exchange ratio as modelling the different engagements and strategies by either side that result in deviations from the historic exchange ratio and the general uncertainty in warfare commonly refered to as the "Fog of War" [Clausewitz and Maude 1982; Herbig, 1986; Waldman, 2010|.

\section{NUMERICAL SIMULATIONS OF THE COMBAT MODEL}

The parameter values used in our numerical investigation are provided on Table 1. Initially, the ground combat power of the attackers and defenders are $A_{0}=350,000$ and $D_{0}=200,000$ respectively. The attackers are assumed to be willing to endure a higher attrition rate than the defenders $(a=0.3$ and $d=0.15$ ). The mean exchange ratio $\rho$ and the initial value of the mean exchange ratio $\rho_{0}$ of attackers lost to defenders is taken to be the historical value of 1.5 as defenders are generally considered to be more effective in combat Epstein [1985]. The value of $\sigma=0.2$ accounts for the size of the variations in the exchange ratio that result as a consequence of the uncertainty of warfare [Clausewitz and Maude, 1982; Herbig, 1986; Waldman, 2010| and the evolving strategies by either side. The value of $\lambda=0.2$ accounts for the speed at which the exchange ratio returns to its historical value $(\rho=1.5)$ in the event of deviations from its mean. Initially the defender is unwilling to yield any ground to the attacker $\left(W_{0}=0\right)$. However once the defender attrition threshold of $d=0.15$ has been exceeded, the defender yields ground by withdrawing up to a maximum rate of $W_{\max }=20 \mathrm{~km}$ per day. For a comparison of the output of this model to the deterministic setting, see Epstein [1985].

Two quantities of interest are the day upon which a side is victorious and the combat power of the winning side once combat ends. The day of victory, or time till victory, is denoted as $\tau$, and is defined by

$$
\tau=\inf \left\{n>0:\left(A_{n} \leq C\right) \cup\left(D_{n} \leq C\right)\right\}
$$


We regard either side as being defeated when their combat power is less than or equal to a critical value $(C \geq 0)$. We take $C=100$ as the point where a side admits defeat and surrenders which then ends the combat. The combat power of the attackers ( or defenders) in the event of victory is given by $A_{\tau}$ ( or $\left.D_{\tau}\right)$.

In Figures $1 \& 2$ we show a single realisation of the cases of an Attacker victory and a Defender victory respectively. In each figure we

\begin{tabular}{c|c|c} 
Parameter/Variable & Description & Value \\
\hline \hline$A_{0}$ & Initial attacker ground combat power/force strength & 350,000 \\
$D_{0}$ & Initial defender ground combat power/force strength & 200,000 \\
$a$ & Attacker attrition rate threshold & 0.3 \\
$d$ & Defender attrition rate threshold & 0.15 \\
$\gamma_{0}$ & Attacker prosecution rate & 0.15 \\
$W_{0}$ & Initial defender withdrawal rate $(\mathrm{km} /$ day $)$ & 0 \\
$W_{\max }$ & Maximum defender withdrawal rate $(\mathrm{km} /$ day $)$ & 20 \\
$\rho$ & Mean exchange ratio & 1.5 \\
$\rho_{0}$ & Initial exchange ratio & 1.5 \\
$\sigma$ & Exchange ratio volatility & 0.2 \\
$\lambda$ & Exchange ratio mean reversion & 0.2 \\
$L_{\rho}$ & Exchange ratio lower bound & 0.1
\end{tabular}

Table 1: Parameter and variable values used in the simulations based on Epstein |1985]. provide the daily values of the Attacker and Defender ground combat power, the attrition rates, the defender withdrawal rate, and the exchange ratio. In the realisation where the attacker wins, Figure 1, the adaptive behaviour of each side is similar throughout the combat period. After the first day the defenders attrition rate exceeds its threshold value and continues to exceed it for the rest of combat, i.e. $\delta_{n}>d$ for $n \in[1, \ldots, \tau]$. As a result, the defenders withdraw every day at an increasing rate. Upon defeat we have $W_{\tau} \approx W_{\max }$. The success of the attackers in this realisation can be inferred from the exchange ratio process which dips well below its mean value after day $n=1$, only returning to a value similar to its mean around day $n=10$. The superior number of forces of the attackers coupled with a favourable exchange relative to the historical nominal value $(\rho=1.5)$ results in victory for the attackers. The impending success of the attackers can be seen as early as day $n=3$ in the two attrition rates. Initially the attackers maintain an attrition rate close to their prosecution rate. However after day $n=3$, the attackers attrition begins to drop as the defenders withdraw at an increasing rate. By the end of combat, when the defenders are withdrawing at a near maximum rate, the attackers attrition is near 0 . In the case of the defenders, their attrition rate at $n=3$ far exceeds their attrition threshold value and it is unable to recover to pre-threshold attrition levels.

In the case where the defenders achieve victory, Figure 2, the adaptive behaviour of the forces plays a larger role then in the previous case of the attackers victory. In the first half of combat, the defenders employ a withdrawal strategy which reduces their attrition rate $\delta_{n}$. There are three distinct periods of withdrawal: from days $n=1, \ldots, 9$; days $n=10, \ldots, 14$; and on day $n=16$. After day 16 the defenders no longer need to withdraw and so directly oppose the attacking force. Due to the defenders strategy, the attackers endure an attrition rate close to or equal to their prosecution rate for the majority of combat. The failure of the attackers to achieve victory can be seen in the exchange ratio process where during the first 11 days of combat $\rho_{n}>\rho$, after which the attackers strength is less than that of the defenders. In this case the defenders choice of strategy, plus the uncertainty of warfare, favours them through a large exchange ratio, meaning that they exceed their expected effectiveness in defending their ground. In both cases the outcome of combat was decided in the first few days through fluctuations in the exchange ratio process. Values of $\rho_{n}<\rho$ for small $n$ favour the attackers whilst values of $\rho_{n}>\rho$ for small $n$ favour the defenders. The significance of an advantage in the initial stages of combat can be observed in the second case where, after day $n=10$, the attackers have several favourable engagements relative to the mean exchange ratio. However these engagements do not make up for the defenders successes in the initial days of combat: the attackers eventually suffer a defeat as the defender ceases to withdraw and directly oppose the attackers to the point of their annihilation.

To investigate the expected results of our model for the parameter set on Table 1 and the impact of a greater volatility in the exchange ratio on the outcome of combat we perform Monte-Carlo simulations [Robert and Casella, 2013| to evaluate statistics of interest. Namely, the probability of an attacker victory, $\mathbf{P}($ Attacker victory $)=\mathbf{P}\left(\left(A_{\tau}>C\right) \cap\left(D_{\tau} \leq C\right)\right)$, the attacker ground force strength in the event that the attacker is victorious, $A_{\tau}$ given $\left(\left(A_{\tau}>C\right) \cap\left(D_{\tau} \leq C\right)\right)$, and the day of victory in the event that the attacker is victorious, $\tau$ given $\left(\left(A_{\tau}>C\right) \cap\left(D_{\tau} \leq C\right)\right)$. For the last two cases, we use a Kernal Density Estimation (KDE) procedure in python to produce the corresponsing densities where the bandwidth is chosen using Silverman's rule [Silverman, 2018|. In Figure 3, we observe two cases for $\lambda=0.2$ and $\lambda=0$ for varying $\sigma \in[0.1,1]$ where the former case corresponds to a mean reverting process with a reflecting boundary at $L_{\rho}$ and the latter to a Gaussian random walking with a reflecting boundary at $L_{\rho}$. 
T. A. McLennan-Smith et al., Stochastic processes in a discrete model of ground combat

(a)

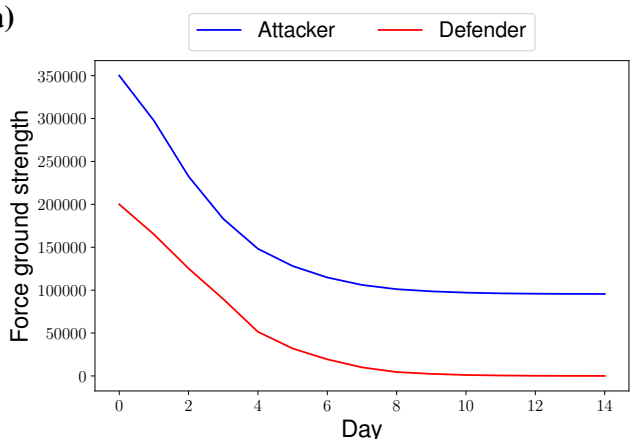

(c)

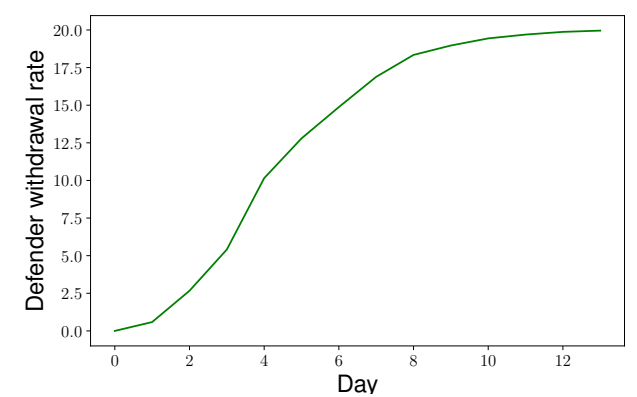

(b)
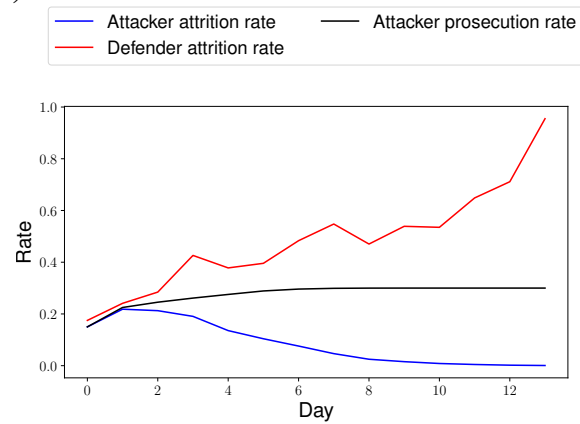

(d)

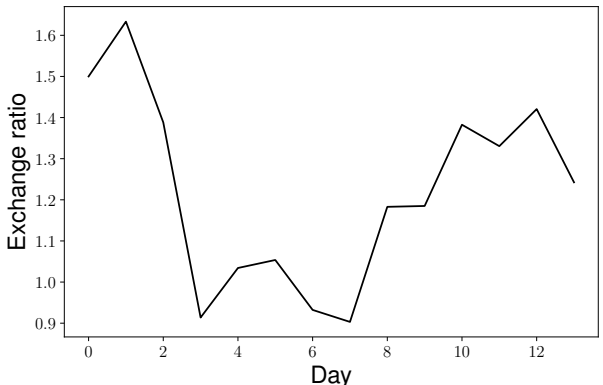

Figure 1. Plots of a single realisation of the model in the case of an Attacker victory. (a) : Time series plots of the attacker and defender ground forces strength, $A_{n}$ and $D_{n}$ respectively. (b) : Time series plots of the attrition rates $\alpha_{n}, \delta_{n}$, and $\gamma_{n}$. (c) : Time series plot of the defender withdrawal rate $W_{n}$. (d) : Time series plot of the exchange ratio $\rho_{n}^{+}$.

(a)

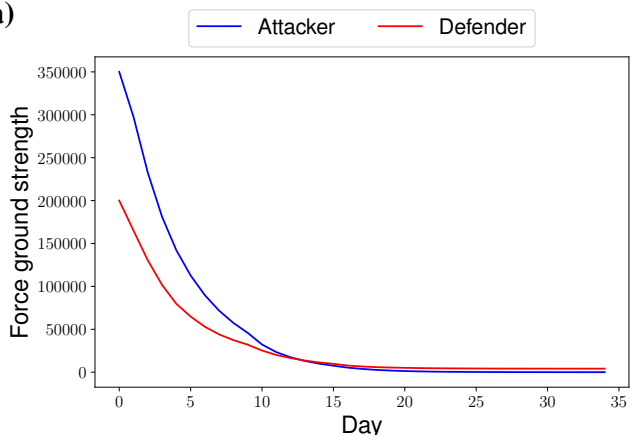

(c)

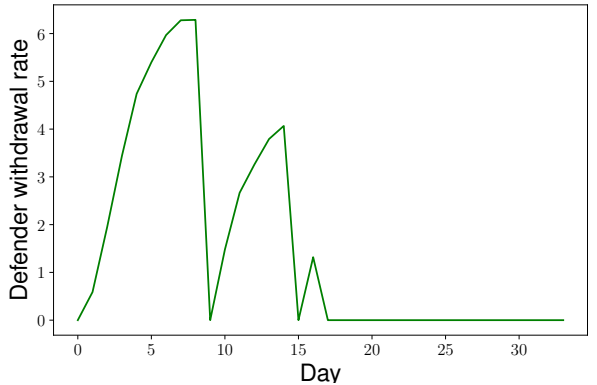

(b)
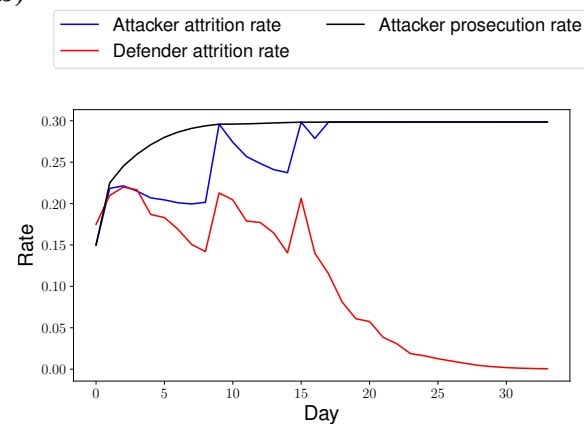

(d)

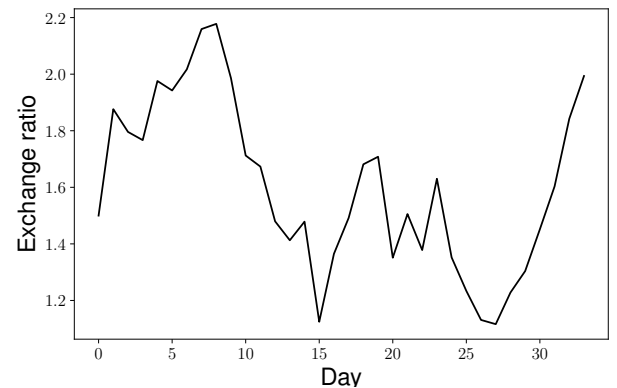

Figure 2. Plots of a single realisation of the model in the case of a Defender victory. (a) : Time series plots of the attacker and defender ground forces strength, $A_{n}$ and $D_{n}$ respectively. (b) : Time series plots of the attrition rates $\alpha_{n}, \delta_{n}$, and $\gamma_{n}$. (c) : Time series plot of the defender withdrawal rate $W_{n}$. (d) : Time series plot of the exchange ratio $\rho_{n}^{+}$. 
Our Monte-Carlo simulations are run with $N=50000$ samples. Observing the plots of the probability of an Attacker victory vs $\sigma$, we notice that the Attacker is generally favoured for small values of $\sigma$ which to be expected as in the deterministic case the attacker is victorious. The probability of success decreases as $\sigma$ increases. However, at large values of $\sigma$ the attackers may obtain an early victory with a larger ground force strength remaining than in the case for small $\sigma$. Noticeably, there is potential for the attackers to achieve victory on day $n=1$ which results in the peak in $f\left(A_{\tau}\right)$ at $A_{\tau} \approx 245,000$ for large $\sigma$. A smaller peak can be also observed in $f\left(A_{\tau}\right)$ at $A_{\tau} \approx 200,000$ for large $\sigma$ as a result of the attackers being victorious on day $n=2$. We can thus view the choice of $\sigma$ as a "risk vs reward" type situation where, in interpreting the size of $\sigma$ as being a result of increasingly divergent and complex attacker strategies from traditional assaults, complex strategies come with an inherent risk to the attacker in increasing its chance for defeat. However the potential reward is that the combat is potentially over sooner and less attacker attrition is suffered as a result. We observe that for $\sigma=0.1, \mathbf{E}\left[A_{\tau}\right] \approx 51,000$ and $\mathbf{E}[\tau] \approx 17.9$ and for $\sigma=1, \mathbf{E}\left[A_{\tau}\right] \approx 110,000$ and $\mathbf{E}[\tau] \approx 9$.

(a)

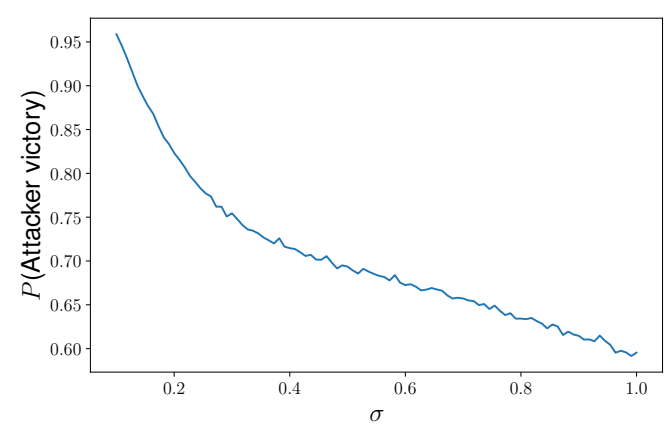

(c)

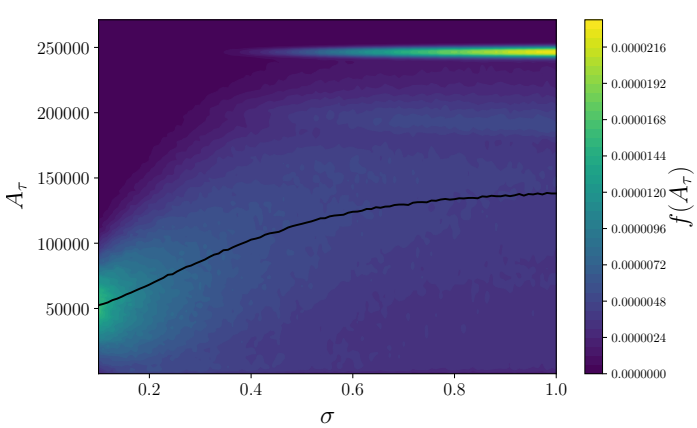

(e)

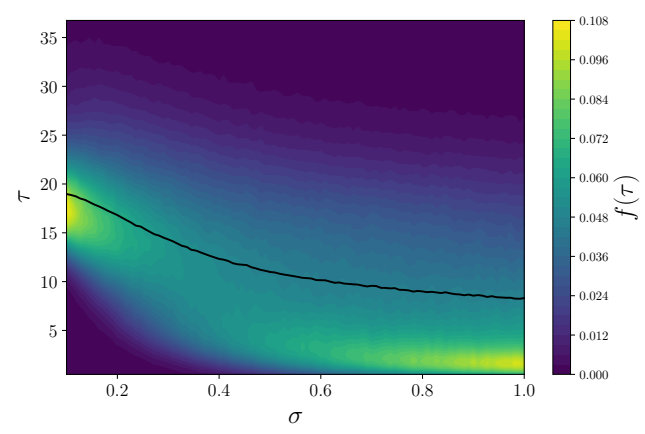

(b)

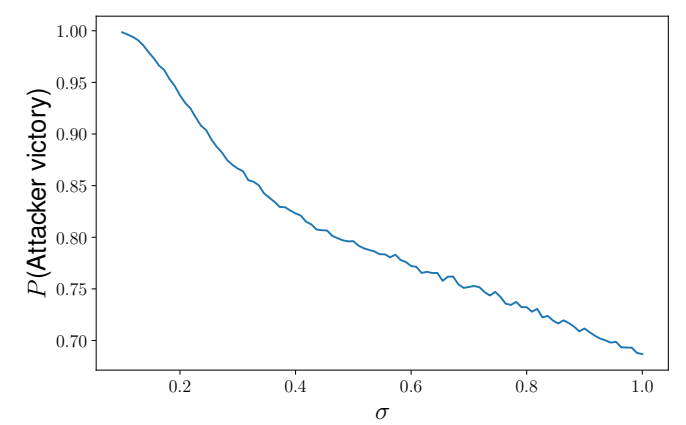

(d)

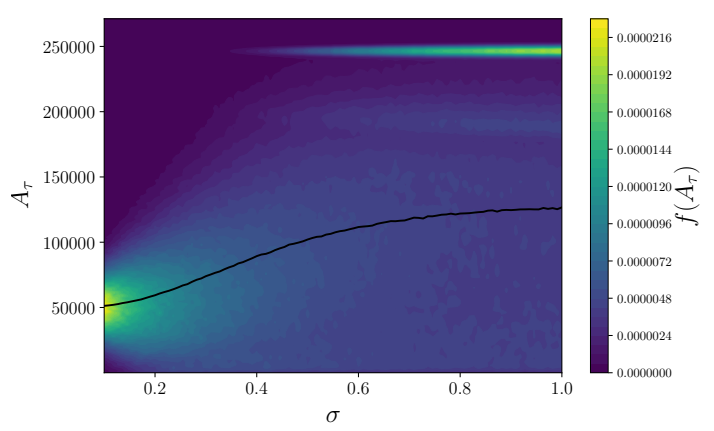

(f)

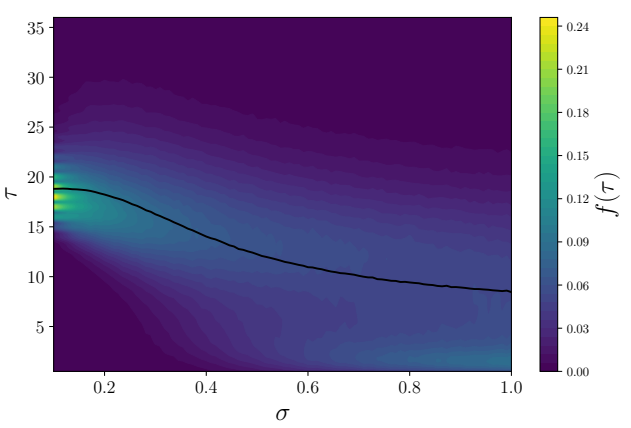

Figure 3. Left column: Monte-Carlo simulations where the exchange ratio is Gaussian random walk with reflecting boundary at $L_{\rho}$ with $\lambda=0$, Right column: Monte-Carlo simulations where the exchange ratio is mean reverting process with a reflecting boundary at $L_{\rho}$ with $\lambda=0.2$. (a) and (b): Probability of attacker victory vs $\sigma$. (c) and (d): Kernal Density Estimates (KDEs) of the remaining attacker forces in the case of victory, $A_{\tau}$ vs $\sigma$, (e) and (f): KDEs of the attacker victory day, $\tau$, vs $\sigma$. 


\section{Discussion And Conclusion}

As our scenario is asymmetric with regards to the nature of the attackers and defenders, our implementation of the exchange ratio is asymmetric in that the process $\rho_{n}^{+}$does not account for the difference in scaling where the attackers are favoured $\rho_{n}^{+} \in\left(L_{\rho}, 1\right)$ and where the defenders are favoured $\rho_{n}^{+} \in(1, \infty)$. Consequently, it is possible when $\sigma$ is large for the attackers to achieve an exchange ratio close to the lower bound, that is $\rho_{n}^{+} \approx L_{\rho}$ for some $n$, however the same is not true for the defenders. We can interpret this part of the asymmetry as the attackers being capable of employing superior firepower that can overwhelm the defenders at minimal cost. Additionally, this implementation has the property that $\mathbf{P}\left(\rho_{n}^{+}>\rho\right)>\mathbf{P}\left(\rho_{n}^{+}<\rho\right)$ for $n \geq 1$ due to the reflecting boundary at $L_{\rho}<\rho$. In other words the defenders will generally perform better than expected when combat uncertainty is introduced through the "Fog of War" and the complex tactics employed by the attackers. However the magnitude of this deviation from the mean is less than that of which the attackers are able to achieve. A more symmetric scaling of the exchange ratio in the sense of attacker or defender favourability can be obtained in Eq 8, where $\rho_{n}^{+}$is now replaced by $\rho_{n}^{(r)}$ and when using the historic exchange ratio of 3:2 [Epstein, 1985], $\rho_{n}^{(r)}$ is a discrete, stochastic process on the domain $\bar{D}=[0, \infty)$ which is defined by

$$
\rho_{n}^{(r)}= \begin{cases}\frac{3+\frac{2}{3} \rho_{n}}{2} & \text { if } \rho_{n} \geq 0 \\ \frac{3}{2-\rho_{n}} & \text { otherwise }\end{cases}
$$

Here $\rho_{n}$ is the mean-reverting stochastic process with $\mathbf{E}\left[\rho_{n}\right]=0$ defined by

$$
\rho_{n+1}=\rho_{n}-\lambda \rho_{n}+\sigma X_{n}, \quad \rho_{0}=0 .
$$

In this implementation, the number of attackers lost to defenders is $\left(3+\frac{2}{3} \rho_{n}\right): 2$ if $\rho_{n}$ is positive and $3:\left(2-\rho_{n}\right)$ if $\rho_{n}$ is negative.

A key feature of our results for varying $\sigma$ is the "risk vs reward" scenario that arises for the attackers. In the deterministic setting the Attackers achieve victory with $A_{\tau} \approx 50000$ : a loss of approximately $85 \%$ of their initial strength. Increasing $\sigma$, which can be interpreted as the attackers taking on more complicated and riskier tactics, has the potential to greatly reward the attackers: the duration of combat may be shorter and the attackers may suffer less attrition. However, this risk comes at the cost that victory is no longer assured.

We have used the parameter set given in Epstein |1985|. Future work may explore the dynamics of the model as these parameters are varied. Possible extensions of the model are to include recruitment and the generalisation of our stochastic process to allow for jump processes to represent sudden shifts in the course of combat.

\section{REFERENCES}

Buontempo, J. T., H. G. Potrykus, and A. I. Kaufman (2006). A symmetric adaptive model of combat. Journal of Mathematical Sociology 30(2), 113-136.

Clausewitz, C. and F. N. Maude (1982). On war. Penguin UK.

Epstein, J. (1985). The calculus of conventional war: dynamic analysis without Lanchester theory.

Epstein, J. M. (1987). Strategy and force planning: The case of the Persian Gulf.

Herbig, K. L. (1986). Chance and uncertainty in on war. The Journal of Strategic Studies 9(2-3), 95-116.

Lancaster, F. (1916). Aircraft in warfare: The dawn of the Fourth Arm. London, England: Constable.

Robert, C. and G. Casella (2013). Monte Carlo statistical methods. Springer Science \& Business Media.

Sedaghat, H. (2002). Convergence, oscillations, and chaos in a discrete model of combat. SIAM review 44(1), 74-92.

Silverman, B. W. (2018). Density estimation for statistics and data analysis. Routledge.

Waldman, T. (2010). Shadows of Uncertainty: Clausewitz's timeless analysis of chance in war. Defence Studies 10(3), 336-368. 